\title{
Video game experience affects performance, cognitive load, and brain activity in laparoscopic surgery training
}

Hasan Onur Keles ( $\square$ hokeles@ankara.edu.tr)

Ankara University https://orcid.org/0000-0001-8493-2582

Ahmet Omurtag

Nottingham Trent University

\section{Research Article}

Keywords: video games, surgery, cognitive load

Posted Date: September 17th, 2021

DOl: https://doi.org/10.21203/rs.3.rs-904919/v1

License: (c) (i) This work is licensed under a Creative Commons Attribution 4.0 International License.

Read Full License 


\section{Abstract}

BACKGROUND Video games can be a valuable tool for surgery training. Individuals who interact or play video games, tend to have a better visuospatial ability when compared to non-gamers. Numerous studies suggest that video game experience is associated with faster acquisition, greater sharpening, and longer retention of laparoscopic skills. Given the neurocognitive complexity of surgery skill, multimodal approaches are required to understand how video game playing enhances laparoscopy skill. METHODS Data from thirteen surgeons with varying levels of laparoscopy experience and no video game experience, and 27 students with no laparoscopy experience and varying levels of video game experience performed standard laparoscopic training tasks. Their performance, subjective cognitive loading, and prefrontal cortical activity were recorded and analyzed. RESULTS The surgeons performed the tasks significantly faster, had significantly lower cognitive load as well as less left prefrontal activation, relative to the nongamer novices. In gamer novices, the task completion time decreased significantly with increasing game experience. The cognitive load decreased significantly with increasing gaming experience in gamer novices and with laparoscopy experience in surgeons. CONCLUSION Our results suggest that along the dimensions of performance, cognitive load, and brain activity, the effects of video gaming experience on novice laparoscopy trainees are similar to those of real-world laparoscopy experience on surgeons. We believe that the neural underpinnings of surgery skill and its links with gaming experience need to be investigated further using wearable functional brain imaging.

\section{Introduction}

Laparoscopic surgery offers substantial benefits to patients, including small incisions, rapid recovery, short hospital stays, and reduced post-operative pain. These translate into increased patient safety and significant economic benefits to healthcare systems. However laparoscopic surgery is difficult and imposes additional demands on surgeons' perceptual and cognitive abilities. A laparoscopic surgeon operates with an indirect, narrow visual access and minimal tactile feedback. Such conditions require new skills with different learning curves and new training methods beyond the traditional masterapprentice format. It is critical for residents to reach expertise in a safe training environment with documented criteria. There is increasing interest in characterising not only the observed performance but also the cognitive effort and physiological and brain activity profiles of trainees with the ultimate aim of devising better training and assessment methodologies $(1-3)$. While direct observation may indicate that a trainee performs adequately, it may fail to predict the long term retention of skill or actual performance beyond the training environment, and trainees who perform identically may differ in subsequent realworld performance.

Video games can be a valuable tool for surgery training. Video gamers demonstrated superior eye-hand coordination, faster reaction times, superior spatial visualization skills, high capacity for visual attention and spatial distribution. Both laparoscopic surgery and computer games require eye-hand coordination, visuospatial cognitive ability, attention and perception skills. Individuals who interact or play video games, tend to have a better visuospatial ability when compared to non-gamers $(4,5)$. Numerous studies 
suggest that video game experience is associated with faster acquisition, greater sharpening, and longer retention of laparoscopic skills (6-11). Video game playing also appears to have value as warm-up preparatory exercise for surgery. These advantages accrue preferentially to laparoscopic or robotic (12) rather than to traditional surgery. Yet, neither the direction of causality nor the mechanisms through which video game experience affects surgery skills are well understood (13). To our knowledge the studies to date which investigated these links exclusively utilized overt performance and behavioural measures. However given the neurocognitive complexity of surgery skill, multimodal approaches are required.

We investigated the impact of video game playing experience on the performance, subjective task load, and prefrontal (PFC) brain activity of novice trainees, and compared them with the corresponding variables in expert surgeons of varying levels of laparoscopy experience. To measure performance we chiefly employed task completion times. For cognitive load we used NASA-TLX which has been widely used to measure subjective task load in surgery (14-16). It is a multidimensional ratings scale that provides an overall index of mental workload as well as the relative contributions of six subscales: mental, physical, and temporal task demands; and effort, frustration, and perceived performance. We also used a high-density, wireless functional near-infrared spectroscopy (fNIRS) device, which allowed us to discover the hemoglobin concentration changes as a proxy for brain function. Results indicated that video game experience was correlated with higher performance and lower cognitive load, and the prefrontal cortical activation of students with gaming experience was relatively lower than those without gaming experience. In terms of these variables gaming experience in novices tended to produce effects similar to those of laparoscopy experience in surgeons.

\section{Methods}

\section{Subjects}

Data from thirteen surgeons with varying levels of laparoscopy experience and no video game experience, and 27 students with no laparoscopy experience and varying levels of video game experience were used in this study. The students were subdivided into 12 non-gamer students (with no experience in video gaming) and 15 gamer students (with experience in video gaming). Subject demographics are listed in Table 1. All participants provided written informed consent at the beginning of the experimental session. Participants had normal or corrected to-normal vision. The Ethical Committee of the College of Medicine at Medipol University (10840098-604.01.01-E.33230) approved the study.

\section{Experimental Design}

Participants performed standard training tasks including peg transfer and string pass using a laparoscopic trainer box. Data were recorded including time to completion, error rate, and overall work quality. After subjects completed each task, they completed the NASA task load index (NASA-TLX) questionnaire. At the beginning of the experiment, two 2-minutes-long videos that demonstrates the tasks were shown on a computer screen to the subject. After the video session the 15 min training was repeated by each subject for gamers and non-gamers. Further details of the procedure were provided in a recent 
study where we reported an analysis of the brain activity of surgeons and a subset of the student participants without regard to game playing experience (3). All participants filled out a questionnaire detailing their video game experience including the frequency, duration and category. The gamers were divided into three groups based on their replies to the questionnaire: Only Shooter game group (that played first-person shooter and/or third person shooter and/or Role playing games), Only Strategy game group (real-time strategy, turn based strategy and multiplayer battle arena games), Shooter + Strategy game group (both strategy and shooter games).

\section{Data Analysis}

Completion times of three laparoscopic tasks were recorded. The participants used a NASA-TLX to evaluate five criteria: mental, physical, and temporal task demands; and effort, frustration, and perceived performance. Each criterion was scored from 1 to 20. A total mental workload score was obtained from the sum of the criteria scores. To analyze the fNIRS data, source-detector pair readings (with a separation of $3.35 \mathrm{~cm}$ ) at two distinct wavelengths are first converted into hemoglobin concentration changes via the modified Beer-Lambert law, and the average of the task episode are calculated (3). In this paper the standard deviation of the oxyhemoglobin concentration changes over an episode is denoted $\mathrm{HbO}$ and represents the extent of local cortical activation of a subject during the corresponding experimental episode.

\section{Statistical analysis}

When conducting a regression analysis comparing 2 numerical variables, linear fit with analysis of variance was used. The descriptive results comparing two groups, such as completion time v game experience; completion time $\mathrm{v}$ laparoscopy experience; NASA total $\mathrm{v}$ game experience and NASA total $\mathrm{v}$ laparoscopy experience, $\mathrm{HbO}$ changes contained non-paired data. In order to assess the statistical significance of the difference between two groups of non-paired results, we used the non-parametric Kolmogorov test. We did not utilize null-hypotheses whose rejection would have required corrections for multiple comparisons or false discovery.

\section{Results}

We present the behavioral and subjective metrics as well as the cortical activations of novice students (non-gamer and gamer) and expert surgeons, measured while performing laparoscopy training tasks. Forty participants (13 surgeons, 27 students, mean age $27.1 \pm 4.8$ years) were enrolled in the study. The participants' demographics, previous experience with laparoscopic surgery and gaming experience are shown in Table 1. Figure 1 compares the task completion time, NASA-TLX score, and the task-evoked oxyhemoglobin changes in the left prefrontal cortex of the three groups of participants. In Fig. 2 and Fig. 3, the inter-group comparisons of performance and cognitive load that were summarized in Fig. 1 are further elaborated within each group. Finally the subject averaged distributions of cortical activation over the PFC of the participants are shown in Fig. 4. 
Figure $1 \mathrm{~A}$ shows that the surgeons completed the task in a significantly shorter period of time when compared to gamers and non-gamers. A significant difference in completion time was noted between surgeons and non-gamers $(P<0.01)$ confirming the difference between the two groups. Although gamers appeared to be faster, the difference between gamers and non-gamers did not reach significance in Task 1. In Task 2 (not shown), there were similar trends although group differences were not statistically significant. Figure 1B shows that the surgeons had lower NASA-TLX workload scores when compared to gamers $(P<0.01)$ and non-gamers $(P<0.04)$ during Task 1 . There was a similar trend for Task 2. Significant difference in task load was seen between gamers and non-gamers $(P<0.02)$. Figure $1 C$ indicates that the left prefrontal cortical activation of surgeons in Task 1 was significantly lower than that of gamers $(P<0.004)$ and non-gamers $(P<0.02)$. As in the previous comparisons, Task 2 differences between groups were similar.

Figure $2 \mathrm{~A}$, through regression analysis we found a significant association between completion time and gaming experience. The extent of game experience was negatively correlated with completion time $\left(\mathrm{R}^{2}=\right.$ $0.22, \mathrm{P}<0.01)$. This figure only presents the results for Task 1 . We observed a similarly significant association in Task $2\left(R^{2}=0.18, P<0.02\right)$. This figure also shows gaming categories which gamers play (the colour coded presentation used to show the gaming categories). No significant different was seen between only shooters player $v$ only strategy players; only shooters player v strategy + shooters players; only strategy players $v$ strategy + shooters player and Game experience. Figure 2B suggests that more experienced surgeons tended to be faster; however laparoscopy experience was not significantly correlated with completion time of task $1\left(R^{2}=0.15 P=0.17\right)$. Similar results were found in Task2.

Figure 3A, we show the NASA-TLX subscales that indicate the distinct dimensions of cognitive load. Surgeon had lower scores of the six subscales of the NASA-TLX questionnaire compared to gamers and non-gamers for Task1. A significant difference in physical $(<0.02)$ and temporal demands $(<0.0007)$ was noted between surgeons and non-gamers during Task 1 . Effort of surgeons was significantly lower compared to gamers $(<0.05)$ in completing the tasks. No significant difference was seen between surgeons, gamers, non-gamers in performance, frustration and mental demand. In Task 2, no significant difference was seen for pairs of groups in the individual subscales. Figure 3B indicates that novice participants with higher game experience reported significantly lower NASA-TLX workload scores $\left(R^{2}=\right.$ $0.21, P<0.001$ ). Similarly (Fig. 3C) we found that more years of laparoscopy experience correlated with significantly lower NASA-TLX workload scores $\left(R^{2}=0.36, P<0.0001\right)$. In Fig. 3B and Fig. 3C, we present the NASA-TLX scores for both Task 1 and Task 2. With regard to the topographic distribution of brain activity in the 3 groups, Fig. 4 indicates that the left prefrontal cortical activation in non-gamers was substantially higher than that in gamers which, in turn, was somewhat higher than that and surgeons.

\section{Discussion}

Our results suggest that some of the effects of video gaming experience on novice laparoscopy trainees are similar to those of real-world laparoscopy experience on surgeons. We have shown this by using 
performance and subjective metrics as well as brain activity. To our knowledge this is the first study that includes cognitive and brain metrics, and not just behavioral ones, in the investigation of video game experience in relation to laparoscopy skill, and directly compares these to those obtained from expert surgeons.

Taking novice students with no laparoscopy or gaming experience as a baseline, Fig. 1 shows that task completion time, self-reported cognitive load, and left PFC activation all decreased with increasing gaming experience or with greater laparoscopy experience. Some of these inter-group differences were statistically significant and all followed a clear trend. Furthermore, Fig. 2A shows that within the group of gamer students, students with greater gaming experience completed the training task significantly faster. In previous studies video game playing correlated with better surgical technique in medical school students and impacted the traditional skills of knot tying, incision making and suturing (9). Evidence shows that first-person shooter games preferentially enhance attentional control and executive function(5), however whether a particular genre of video game plays a particular role has yet to be determined in a randomized controlled study. We have not been seen any differences in the effect of genres of game, likely due to insufficient data. In addition to the effect of game playing on performance, we have shown in Fig. 2B that the number of laparoscopy procedures performed in the past may have enhanced surgeons' task performance.

Figure 3 suggests that there was a significant negative correlation between the cognitive load and gaming experience in students and laparoscopy experience in surgeons. This result it significant since NASA-TLX scores have been shown to independently predict future OR performance (14). In addition higher cognitive load during surgery may lead to distraction, consideration of fewer options than those available, or inflexibility in choosing strategies. Low load on the other hand allows greater amounts of data to be processed, leading to appropriate responses to unexpected events (17).

Figure 1 and Fig. 4 suggest that PFC activation provided that clearest inter-group discrimination, particularly with regard to differences between non-gamer and gamer novices. Comparison of Fig. 4A and Fig. 4B shows that activity in the left PFC, in particular, is reduced as a result of greater gaming or laparoscopy experience. The reduction in activity is consistent with the known dominance of the left hemisphere in motor action regardless of handedness $(18)$, interference processing $(19,20)$ and overall bi-manual coordination $(21,22)$. By contrast PFC lateralization is reduced in PTSD and other disorders (23). Previous fNIRS studies of laparoscopy skill have indicated that greater skill is accompanied by behavioral automation, which tends to reduce the engagement of the executive areas in the PFC(24).

Our study had a number of limitations including the following.

- Low number of participants. A greater number of participants would allow us to rule out or show the significance of some of the trends observed. It may also reveal the differential effects of the genre of video game. 
- Student participants did not all play the same set of games. More controlled gaming experience may help increase the accuracy and validity of the results.

- Our results show only correlation and not causation. Underlying factor (such as higher dexterity or motivation) could be causing both higher gaming experience as well as better laparoscopy training performance. This shortcoming may be circumvented by an interventional study that manipulates gaming experience systematically.

- The quantification of video game experience may have been subject to inaccuracy since it was selfreported. Both in gaming and laparoscopy experience some self-reporting inaccuracy is evident from the fact that there appears to be a bias to report in multiples of fixed values.

\section{Conclusion}

Our results indicate that multimodal investigations may shed light on how video games affect not only performance in surgery training, but cognitive load and brain activity, as well. The understanding of the neural underpinnings of surgery skill and its links with gaming experience will likely increase in the near future as wearable functional brain imaging becomes more widely available.

\section{Declarations}

\section{Competing Interests:}

The authors have declared that no competing/commercial interests exist

\section{References}

1. Modi HN, Singh H, Yang GZ, Darzi A, Leff DR (2017) A decade of imaging surgeons' brain function (part I): Terminology, techniques, and clinical translation. Surgery 162(5):1121-1130

2. Zakeri Z, Mansfield N, Sunderland C, Omurtag A (2020) Physiological correlates of cognitive load in laparoscopic surgery. Sci Rep 10(1):12927

3. Keles HO, Cengiz C, Demiral I, Ozmen MM, Omurtag A (2021) High density optical neuroimaging predicts surgeons's subjective experience and skill levels. PLoS One 16(2):e0247117

4. Nahum M, Bavelier D (2020) Video games as rich environments to foster brain plasticity. Handb Clin Neurol 168:117-136

5. Green CS, Bavelier D (2012) Learning, attentional control, and action video games. Curr Biol 22(6):R197-R206

6. Rosser JC Jr, Lynch PJ, Cuddihy L, Gentile DA, Klonsky J, Merrell R (2007) The impact of video games on training surgeons in the 21st century. Arch Surg 142(2):181-186; discusssion 6

7. Ou Y, McGlone ER, Camm CF, Khan OA (2013) Does playing video games improve laparoscopic skills? Int J Surg 11(5):365-369 
8. Lynch J, Aughwane P, Hammond TM (2010) Video games and surgical ability: a literature review. J Surg Educ 67(3):184-189

9. de Araujo TB, Silveira FR, Souza DL, Strey YT, Flores CD, Webster RS (2016) Impact of video game genre on surgical skills development: a feasibility study. J Surg Res 201(1):235-243

10. Datta R, Chon SH, Dratsch T, Timmermann F, Muller L, Plum PS et al (2020) Are gamers better laparoscopic surgeons? Impact of gaming skills on laparoscopic performance in "Generation Y" students. PLoS One 15(8):e0232341

11. Chalhoub E, Tanos V, Campo R, Kesrouani A, El Rassy E, Rizkallah J, Chalhoub D, Walter C, Sleiman Z (2016) The role of video games in facilitating the psychomotor skills training in laparoscopic surgery. Gynecol Surg 13:419-424

12. Hvolbek AP, Nilsson PM, Sanguedolce F, Lund L (2019) A prospective study of the effect of video games on robotic surgery skills using the high-fidelity virtual reality RobotiX simulator. Adv Med Educ Pract 10:627-634

13. Jalink MB, Goris J, Heineman E, Pierie JP, ten Cate Hoedemaker HO (2014) The effects of video games on laparoscopic simulator skills. Am J Surg 208(1):151-156

14. Yurko YY, Scerbo MW, Prabhu AS, Acker CE, Stefanidis D (2010) Higher mental workload is associated with poorer laparoscopic performance as measured by the NASA-TLX tool. Simul Healthc 5(5):267-271

15. Miranda G, Casmiro M, Cavassi G, Naspetti R, Miranda E, Sacchetti R et al (eds) Comparative Analysis of Subjective Workload in Laparoscopy and Open Surgery Using NASA-TLX2019; Cham: Springer International Publishing

16. Hart SG. Nasa-Task Load Index (NASA-TLX); 20 Years Later. Proceedings of the Human Factors and Ergonomics Society Annual Meeting. 2006;50(9):904-8

17. Carswell CM, Clarke D, Seales WB (2005) Assessing mental workload during laparoscopic surgery. Surg Innov 12(1):80-90

18. Serrien DJ, Sovijärvi-Spapé MM (2016) Manual dexterity: Functional lateralisation patterns and motor efficiency. Brain Cogn 108:42-46

19. Zhang L, Sun J, Sun B, Luo Q, Gong H (2014) Studying hemispheric lateralization during a Stroop task through near-infrared spectroscopy-based connectivity. J Biomed Opt 19(5):57012

20. Proverbio AM, Azzari R, Adorni R (2013) Is there a left hemispheric asymmetry for tool affordance processing? Neuropsychologia 51(13):2690-2701

21. Rushworth MF, Krams M, Passingham RE (2001) The attentional role of the left parietal cortex: the distinct lateralization and localization of motor attention in the human brain. $\mathrm{J}$ Cogn Neurosci 13(5):698-710

22. Jäncke L, Peters M, Himmelbach M, Nösselt T, Shah J, Steinmetz H (2000) fMRI study of bimanual coordination. Neuropsychologia 38(2):164-174 
23. Tian F, Yennu A, Smith-Osborne A, Gonzalez-Lima F, North CS, Liu H (2014) Prefrontal responses to digit span memory phases in patients with post-traumatic stress disorder (PTSD): a functional near infrared spectroscopy study. Neuroimage Clin 4:808-819

24. Nemani A, Yücel MA, Kruger U, Gee DW, Cooper C, Schwaitzberg SD et al (2018) Assessing bimanual motor skills with optical neuroimaging. Science Advances 4(10):eaat3807

\section{Table}

TABLE 1. Group Demographics

\begin{tabular}{|c|c|c|c|c|c|c|c|}
\hline Group & Number & $\begin{array}{l}\text { Median } \\
\text { Gaming } \\
\text { Hours } \\
\text { Per } \\
\text { Week } \\
\text { (Range) }\end{array}$ & $\begin{array}{l}\text { Gaming } \\
\text { Hours Per } \\
\text { Week } \\
\text { Mean } \pm S D\end{array}$ & $\begin{array}{l}\text { Median } \\
\text { Age } \\
\text { (Range) }\end{array}$ & $\begin{array}{l}\text { Age } \\
\text { Mean } \pm S D\end{array}$ & $\begin{array}{l}\text { Median } \\
\text { LSE In } \\
\text { Number } \\
\text { (Range) }\end{array}$ & $\begin{array}{l}\text { LSE } \\
\text { In Number } \\
\text { Mean } \pm S D\end{array}$ \\
\hline $\begin{array}{l}\text { Non-Gamer } \\
\text { Students }\end{array}$ & 12 & $0(0-0)$ & $0 \pm 0$ & $\begin{array}{l}26.5 \\
(18-30)\end{array}$ & $24.9 \pm 4.4$ & $0(0-0)$ & $0 \pm 0$ \\
\hline $\begin{array}{l}\text { Gamer } \\
\text { Students }\end{array}$ & 15 & $15(1-40)$ & $14 \pm 12.7$ & $\begin{array}{l}19(18- \\
32)\end{array}$ & $20.9 \pm 3.7$ & $0(0-0)$ & $0 \pm 0$ \\
\hline $\begin{array}{l}\text { Laparoscopic } \\
\text { Surgeons }\end{array}$ & 13 & $0(0-0)$ & $0 \pm 0$ & $\begin{array}{l}35(27- \\
50)\end{array}$ & $35.5 \pm 6.3$ & $\begin{array}{l}75(5- \\
350)\end{array}$ & $116 \pm 118.3$ \\
\hline
\end{tabular}

LSE: laparoscopic surgery experience

\section{Figures}



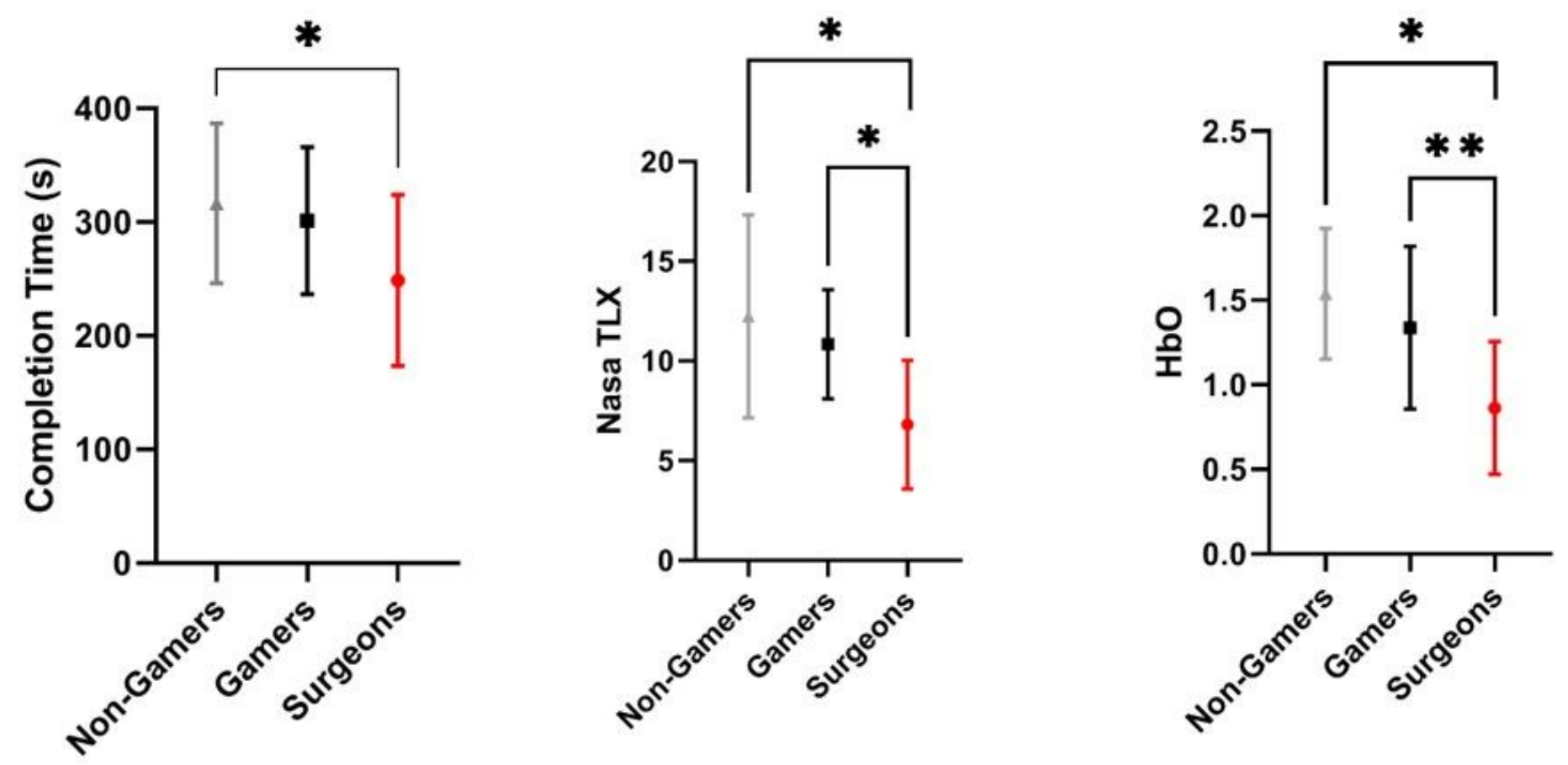

Figure 1

Performance, cognitive load, and prefrontal cortical activations of non-gamer students (gray triangle indicates the median). gamer students (black squares) and surgeons (red circles). The students have no laparoscopy experience and the surgeons have no gaming experience. Error bars indicate sample standard deviations ( ${ }^{*} \mathrm{p}<0.05 ;{ }^{*} \mathrm{p}<0.01$ ). (A) Task Completion Time. (B) Mean NASA-TLX Score. (C) Oxygenated hemoglobin oxyhemoglogin concentration changes in the left prefrontal cortex measured by fNIRS.
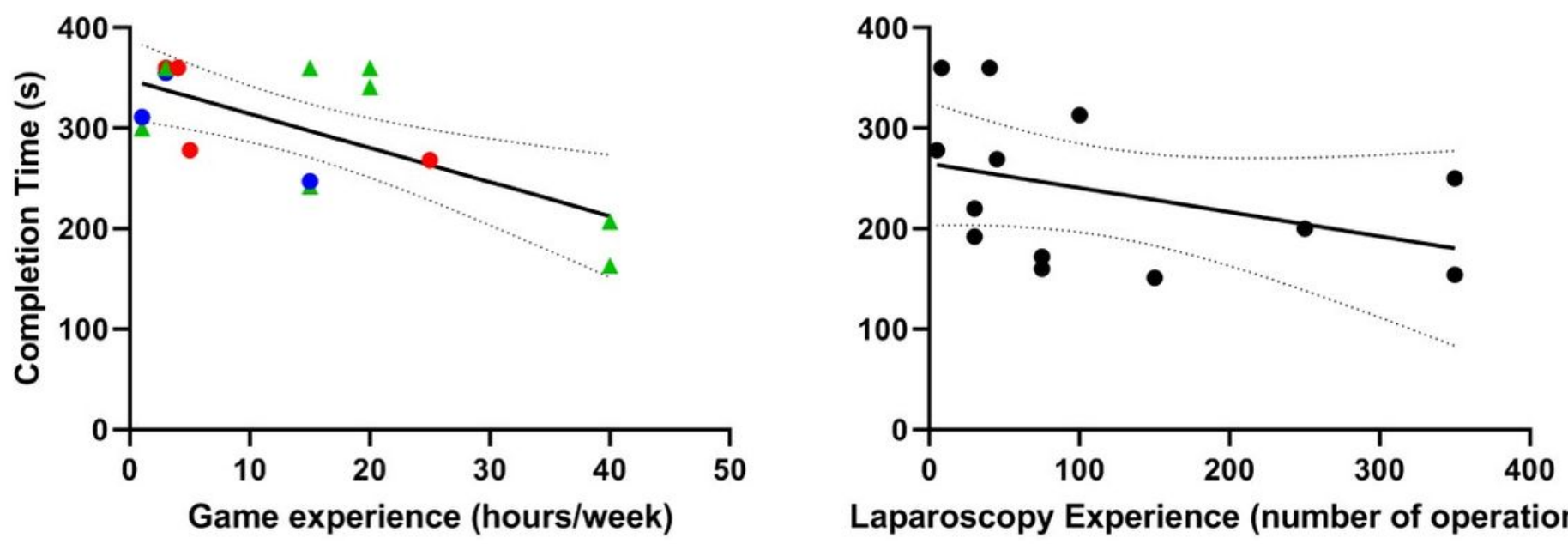

Laparoscopy Experience (number of operations)

Figure 2

Performance and its dependence on the gaming experience of students and on laparoscopy experience of surgeons. (A) Completion Time v gaming experience for students. Gamers are shown as only strategy game players (red circles), only shooter game players (green triangle) and both strategy and shooter 
game players (blue circles). Best fit line to the gamer data is shown ( $R 2=0.22, P<0.01$ ). (B) Completion Time $v$ laparoscopy experience for surgeons. The solid black line indicate the linear best fit and the dotted lines indicate the $95 \%$ confidence interval $(R 2=0.15, P=0.17)$.
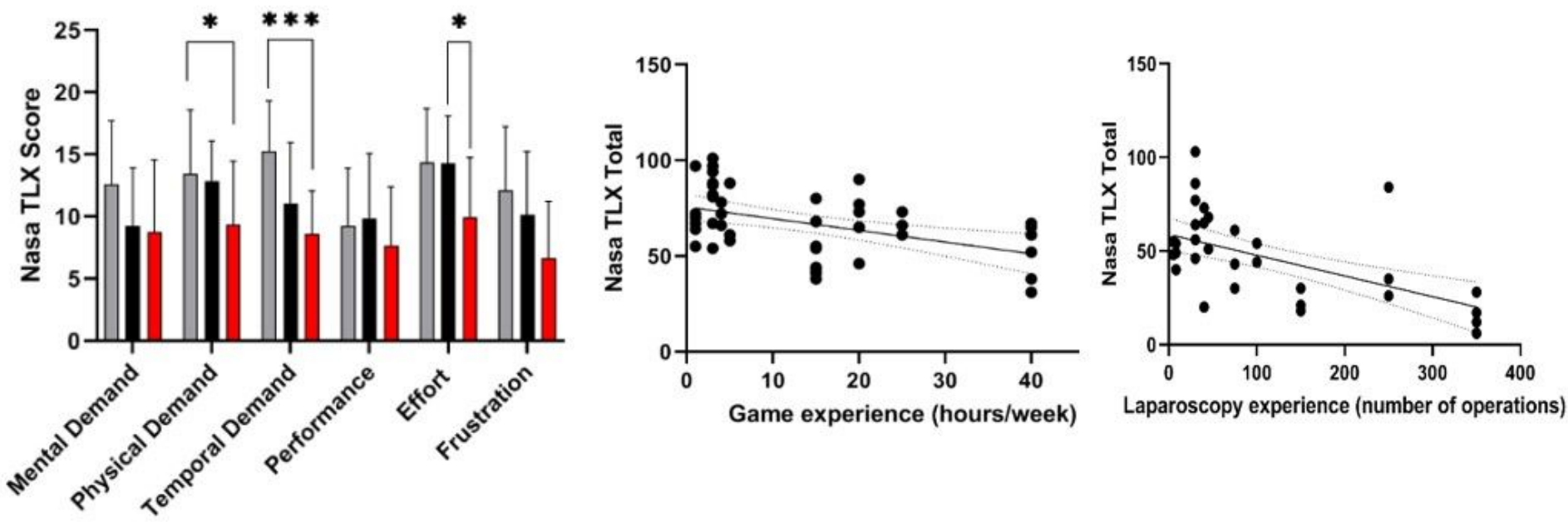

\section{Figure 3}

Cognitive load and its dependence on the gaming experience of students and on laparoscopy experience of surgeons ( $\left.{ }^{\star} \mathrm{p}<0.05 ;{ }^{* \star *} \mathrm{p}<0.001\right)$. (A) Individual dimensions of cognitive load shown by the Nasa-TLX subscores for non-gamer students (gray), gamer students (black), and surgeons (red). Error bars indicate sample standard deviation (B) Total NASA-TLX score $v$ gaming experience for students. $(R 2=0.21$, $P<0.001)$. (C) Total NASA-TLX score $v$ laparoscopy experience for surgeons $(R 2=0.36, P<0.0001)$. In the scatter plots, the solid black lines indicate the linear best fit to the data points and the dotted lines indicate the $95 \%$ confidence interval.
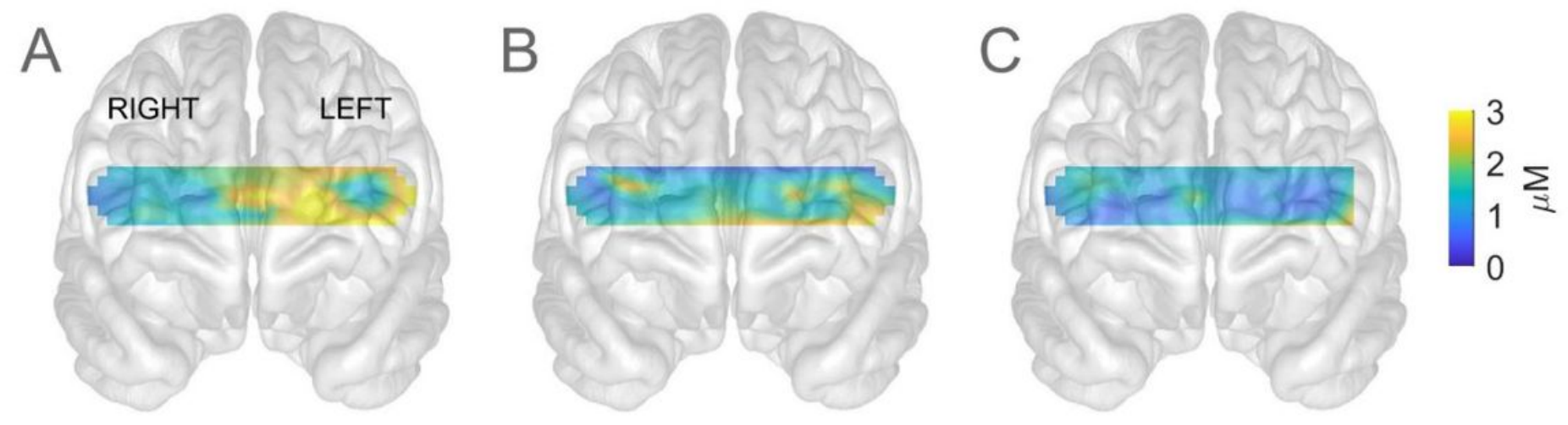

\section{Figure 4}

Frontal view of the task-evoked cortical activations measured by fNIRS. The subject averaged topographic distributions of oxyhemoglobin over the prefrontal cortex are shown. (A) Non-gamer students. (B) Gamer students. (C) Surgeons. 